\title{
Seroprevalencia de brucelosis en trabajadores agrícolas de las comarcas coster as de Castellón, España
}

José Luis Villamarín-Vázquez, MD, (1) Francisco Chiva-N ebot,MD, ${ }^{(1)}$ Alberto Arnedo-Pena, MD.(1)

\author{
Villamarín-Vázquez JL, Chiva-Nebot F, \\ Arnedo-Pena A. \\ Seroprevalencia de brucelosis \\ en trabajadores agrícolas de las comarcas \\ costeras de Castellón, España. \\ Salud Publica Mex 2002;44:137-139.
} El texto completo en inglés de este artículo está disponible en: http://www.insp.mx/salud/index.html

\section{Resumen}

Objetivo. D eterminar la seroprevalencia de brucelosis en trabajadores agrícolas. Material y métodos Estudio transversal efectuado durante 1996 y 1997 en la Unidad de Salud Laboral del Centro de Salud Pública (CSP) de Castellón, España, con 482 aspirantes a su acreditación como manipulador de plaguicidas para aplicación agrícola y a quienes se les hicieron las pruebas serológicas de rosa de Bengala, seroaglutinación deW right y C o mbs antibrucela. En el análisis estadístico se estimó la prevalencia y se usó regresión logística. Resultados 15 personas (3.1\%, IC 95\% 1.8\%-5.1\%) presentaron títulos de 1/40 o mayores a las pruebas de W right 0 Coombs, y todas negativas al rosa de Bengala. $\mathrm{N} 0$ se apreció riesgo profesional elevado. Conclusiones. La prevalencia estimada fue baja. El texto completo en inglés de este artículo está disponible en:http://www.insp.mx/salud/ index.html

Palabras clave: brucelosis;serodiagnóstico; trabajadores agrícolas; España

\author{
Villamarín-Vázquez JL, Chiva-Nebot F, \\ Arnedo-Pena A. \\ Seroprevalence of brucellosis \\ in agricultural workers living \\ in coastal areas of Castellon, Spain \\ Salud Publica Mex 2002;44:137-139. \\ The English version of this paper \\ is available at: http://www.insp. mx/salud/index.html
}

\begin{abstract}
A bstract
Objective. To estimate the seroprevalence of brucellosis in agricultural wo rkers. Material and Methods This crosssectional study was conducted between 1996 and 1997 at Unidad de Salud Laboral del Centro de Salud Pública (CSP) in Castellon, España (Public Health Center O ccupational Health U nit), among 482 subjects applying for certification to handle pesticides for agricultural use. Serologic testing consisted of rose Bengal staining,W right's agglutination, and antibrucella Coombs tests. Statistical analysis consisted in estimation of prevalence figures and logistic regression modelling. Results Fifteen subjects (3.1\%, 95\% Cl 1.8\%-5.1\%) had titers of $1 / 40$ or higher to W right and/or C oombs tests; all were negative to rose Bengal staining. No high occupational risk was observed. Conclusions. A low prevalence of brucellosis was found.The English version of this paper is available at: $h t t p: / / w w w . i n s p . m x / s a l u d / i n d e x . h t m l$
\end{abstract}

Key words: brucellosis; serodiagnosis; agriculture workers; Spain a brucelosis, zoonosis que afecta a varias regiones del mundo, ${ }^{1}$ existe en zonas rurales de España. Pese a su disminución en los últimos años, se declaró una media anual de unos 2000 casos nuevos en el pe- riodo 1995-1999² (tasa de 5 por 100000 habitantes), si bien, hay que considerar el habitual subregistro estadístico. Igualmente, se producen brotes epidémicos por consumo de queso sin pasterizar $^{3} \mathrm{y}$ en trabajadores de

(1) Centre de Salut Publica de Castello, Conselleria de Sanitad, Generalitat Valenciana, España.

Fecha de recibido: 14 de mayo de 2001 - Fecha de aprobado: 1 de diciembre de 2001 Solicitud de sobretiros: Dr.Alberto Arnedo. C/L'0 livera 5, 2-C, 12005-Castellón España. Centro de Salud Pública. Avenida del Mar 12, 12003 Castellón, España.

Correo electrónico: alberto.arnedo@ sanidad.m400.gva.es 
mataderos ${ }^{4}$ lo cual indica que la enfermedad no se encuentra bajo control y que se está lejos de su eliminación. El objetivo de este estudio es determinar la seroprevalencia de brucelosis en trabajadores agrícolas de las comarcas costeras de Castellón, España.

\section{Material y métodos}

Se diseñó un estudio transversal que se desarrolló durante los años de 1996 y 1997 en la Unidad de Salud Laboral del Centro de Salud Pública (ESP) de Castellón, España. Se tomó como población para la muestra a los participantes en el curso para obtener el carnet de manipulador de plaguicidas para uso agrícola, que realiza el CSP y en el cual se incluye un análisis de sangre a cada candidato. En el Laboratorio de Salud Pública del CSP se realizaron tres pruebas serológicas: ${ }^{5}$ rosa de Bengala (RB), seroaglutinación de Wright (SAT), y prueba de Coombs anti-Brucella (PC), empleando como antígeno B. abortus. Para SAT y PC se titularon los sueros empezando por $1 / 10$, con positividad a partir de $1 / 40 .{ }^{6}$ Además, se efectuó una encuesta autocumplimentada por los participantes sobre factores de riesgo de brucelosis. El análisis estadístico de los datos se realizó con el programa Stata. ${ }^{7}$

\section{Resultados}

Realizaron los cursos de manipulador de plaguicidas 547 personas y de ellas participaron en el estudio 482 $(88.1 \%)$, con una edad media de $41.0 \pm 14.0$ años, rango $17-73$, varones $89.1 \%$. La mayoría residían en las comarcas costeras de Castellón (La Plana y El Baix Maestrat) y su ocupación era la agricultura. Nueve $(2 \%)$ habían sufrido brucelosis con anterioridad y 14 (6.3\%) tenían una historia familiar de brucelosis.

Todos los participantes fueron negativos al RB y 15 (3.1\% IC 95\% 1.8\%-5.1\%) tenían títulos de 1/40 o mayores a SAT o PC (cuadro I). Sólo una persona presentó títulos de 1/80 (PC), y 1/40 (SAT), quien meses atrás padeció un proceso febril sin diagnóstico etiológico definitivo. Fueron negativos todos los participantes con historia de brucelosis. Sobre los factores de riesgo, se destacaron los consumos ocasionales de leche no procedente de central lechera (5.5\%), y de queso fresco $(9.4 \%)$. Hubo una baja frecuencia de contacto con cabras y ovejas (1.3\%). Según un modelo de regresión logística, ajustado por edad y sexo, la prevalencia de anticuerpos brucelósicos se asoció con el consumo de leche no procedente de central lechera (OR 9.09; IC 95\% 0.14-180.19) y con el consumo de queso fresco (OR 2.76; IC 95\%:0.47-11.0), pero sin alcanzar significación, $p=0.17$ y $p=0.13$, respectivamente.

\section{Cuadro I Seroprevalencia de anticuerpos anti-brucella en TRABAJADORES AGRICOLAS DE LAS COMARCAS COSTERAS de Castellón, España, 1996-1997}

Seroaglutinación de W right $\begin{array}{lllll}0 & 1 / 10 & 1 / 20 & 1 / 40 & \text { Total }\end{array}$

Prueba de Coombs anti-brucella

\begin{tabular}{lrrrrr}
0 & 438 & 0 & 2 & 1 & 441 \\
\hline $1 / 10$ & 12 & 3 & 0 & 2 & 17 \\
\hline $1 / 20$ & 9 & 1 & 2 & 0 & 12 \\
\hline $1 / 40$ & 8 & 0 & 1 & 2 & 11 \\
\hline $1 / 80$ & 0 & 0 & 0 & 1 & 1 \\
Total & & & & & \\
& 467 & 4 & 5 & 6 & 482 \\
\hline
\end{tabular}

\section{Discusión}

La seroprevalencia de brucelosis fue baja, con un riesgo limitado y esto concuerda con la esporádica notificación de casos de brucelosis en las comarcas estudiadas, en contraste con las del interior montañoso, donde la incidencia es mayor. En España no se dispone de encuestas nacionales de seroprevalencia de brucelosis, y si comparamos con estudios puntuales recientes, la seroprevalencia estimada fue superior a la obtenida, con las mismas pruebas y el antígeno brucelar, en La Cerdanya (Girona) sobre 196 personas, 2.55\% de positivos en población general. ${ }^{6}$ En la comarca del Pallars Jussà (Lleida), Serra y colaboradores, con la prueba de ELISA IgG contra Brucella con antígeno de B. melitensis, obtuvieron una seroprevalencia de $11.9 \%$ sobre población general (346 personas) en zona endémica de brucelosis. ${ }^{8}$ Existen estudios internacionales de seroprevalencia de brucelosis en población general, aunque las comparaciones son problemáticas debido a las pruebas y antígenos empleados en cada caso, y por la definición de caso. En México, se halló una seroprevalencia de $3.42 \%$ (rango $0.24 \%$ - $13.5 \%$ ) en una encuesta nacional con 66982 personas, y en la cual se practicó la microaglutinación en placa (título positivo $\geq 1 / 20$ ) con antígeno B. abortus. ${ }^{9}$ En dos regiones de Italia, se estimó ${ }^{10}$ una prevalencia de 3.1\% en 1294 sujetos de la población general mediante microaglutinación (título positivo $\geq 1 / 40$ ); en zonas endémicas, la prevalencia es más elevada, como en Arabia Saudita, donde $7.8 \%$ de 537 mujeres embarazadas resultaron positivas a una aglutinación a partir de 1/160 (antígenos: B. melitensis y B. abortus) ${ }_{1}^{11}$ en poblaciones de alto riesgo ocupacional por su contacto directo con animales infectados (carniceros, granjeros, matarifes, veterinarios, etcétera), se alcanzó $57 \%, 61 \%$, y 26\% me- 
diante ELISA IgG, IgM, e IgA, respectivamente, sobre 597 personas en el Líbano, con B. abortus como antígeno. ${ }^{12}$ Se emplearon tres pruebas serológicas clásicas complementarias, ${ }^{13}$ con sensibilidades inferiores a las de ELISA y a la intradermo-reacción de la melitina y se detectaron inmunoglobulinas anti-brucella producidas en el curso de la enfermedad; así, IgM por SAT, IgG, esencialmente, IgM e IgA por RB, e IgA y a veces IgG incompletos por PC. ${ }^{14-16}$ No resultaron positivas las personas con historia de brucelosis, y los factores de riesgo detectados no llegaron a ser significativos. Pese a la elevada sensibilidad del RB y su buena correlación con la SAT, 17,18 en pacientes con brucelosis aguda, no hubo positivos al RB. Los participantes no presentaban clínica de brucelosis y 11 tenían títulos de $1 / 20$ o 1/40 para SAT. Lucero y Bolpe, ${ }^{19}$ en Argentina en 307 personas asintomáticas, sólo hallaron una positiva al RB y 13 con títulos de $1 / 25$ o $1 / 50$ para SAT. Al dejar de tener contacto con la bacteria, los niveles de anticuerpos disminuyen con el tiempo y declinan las sensibilidades de RB y SAT.

Serra y colaboradores ${ }^{8}$ indican que técnicas como la ELISA resultarían apropiadas en estudios poblacionales para detectar riesgos actuales o pasados, ${ }_{1}^{14}$ pero éstas no están al alcance de muchos laboratorios. Las pruebas serológicas clásicas son útiles en brotes epidémicos y para detectar brucelosis activa en colectivos de alto riesgo. ${ }^{20,21}$ Hoy, estudios seroepidemiológicos poblacionales permitirían conocer mejor la brucelosis y así facilitar su control y prevención.

\section{Referencias}

1. Corbel MJ. Brucellosis:An overview. Emerg Infect D is 1997;3:213-221. 2. Anónimo. Comentario epidemiológico de las enfermedades de declaración obligatoria y del sistema de información microbiológica. Bol Epidemiol Sem 2000:8:1-12.

3. Castell J, Rúllan JV, Peiró EF, N ieto-Sandoval A. Estudio de un brote epidémico de brucelosis consecutivo al consumo de queso fresco sin pasterizar. Rev Esp Salud Publica 1996;70:303-311.
4. Luna A, Rodríguez A, Suárez T. Análisis de un brote epidémico de brucelosis en trabajadores de un matadero. Rev Esp Salud Publica 1998;72: 137-146.

5.0 rganización Mundial de la Salud. Comité Mixto FAO /O MS de Expertos en Brucelosis. 60 informe. G inebra: 0 rganización Mundial de la Salud, 1986.

6. Subirats E,Vila M,VilaT, Margalef N . Seroprevalencia de brucelosis en La Cerdanya. Med Clin (Bar) 1995;105:48.

7. Stata Corporation. Stata reference manual: Release $3.1 .6^{\text {th }}$ ed. College Station Texas: Stata Corporation, 1993.

8. Serra J, Pujol R, Godoy P. Estudio seroepidemiológico de la brucelosis en un área rural endémica. Enferm Infecc Microbiol C lin 2000;18:74-78. 9. López-Merino A, Migranas-O rtiz R, Pérez-Miravete A, Magos C, Salvatierra-Izaba B,Tapia-C onyer $R$ et al. Seroepidemiología de la brucelosis en México. Salud Publica Mex 1992;32:230-240.

10.Torre I, Ribera G, Pavia M,Angelillo IF.A seroepidemiologic survey on brucellosis antibodies in Southern Italy. Infection 1997;25:150-153.

11. Sharif $A$, Reyes $Z$, Thomassen P. Screening for brucellosis in pregnant women. J Trop Med Hyg 1990;93:42-43.

12.Araj GF, Azzam RA. Seroprevalence of brucella antibodies among persons in high-risk occupation in Lebanon. Epidemiol Infect 1996; 117:281-288.

13. Foz A, Díaz R. Brucelosis. En: Perea EJ, ed. Enfermedades infecciosas. Barcelona: Salvat Editores, 1983:705-725.

14. Piffaretti JC, Staedler P, Beretta-Piccoli C F. Risk of infection by Brucella melitensis for people living near infected go ats. J Infect 1987;15:177-181. 15. Foulton G, Adriambololona L, N guyen BK, Durrande JB, Roux J, Martin-Bouyer G. Epidémiologie des brucelloses. Essai d'évaluation de I'incidence des formes cliniques et infracliniques. Rev Epidem Sante Publ 1981;29:389-398.

16.Bertrand A. Brucellose. Encycl Méd Chir. Paris: Maladies infectieuses, $19818038 \mathrm{~A}^{10,5}$.

17. Colmenero JD, Reguera JM, Cabrera FP, Hernández S, Porras J, Manchado $P$ et al. Empleo combinado del rosa de Bengala e inmunofluorescencia indirecta en el diagnóstico de la brucelosis. Enferm Infecc Microbiol Clin 1989;7:316-320.

18. N avarro A, Pachon J,Torronteras R, C uello JA,Viciana P, López L et al. Utilidad del test de inmunofluorescencia indirecta y la prueba del Rosa de Bengala en el diagnóstico de la brucelosis. Rev Clin Esp 1984;175:27-31. 19. Lucero N, Bolpe J. Buffered plate antigen test as a screening test for diagnosis of human brucellosis. J C lin Microbiol 1998;36:1425-1427.

20. Mishal J, Ben-Israel N , Levin Y, Sherf S, Jafari J, Embon E et al. Brucellosis outbreak: Analysis of risk factors and serologic screening. Int I Mol Med 1999;4:655-658.

21. A bramson 0, Rosenvasser Z, Block C, D agan R. Detection and treatment of brucellosis by screening a population at risk. Pediatr Infect $D$ is J 1991;10:434-438. 